\title{
Diets of deep-water pandalid shrimps on the Western Mediterranean slope
}

\author{
J. E. Cartes \\ Institut de Ciències del Mar, Passeig Nacional s/n, E-08039 Barcelona, Spain
}

\begin{abstract}
The feeding habits of 3 deep-water species of pandalid shrimps occurring in the Catalan Sea (Western Mediterranean) were studied. All specimens were collected from 1988 to 1990 using bottom trawls at depths ranging between 380 and $1249 \mathrm{~m}$. A total of 71 specimens of Plesionika edwardsi, 176 of $P$. martia, and 213 of $P$. acanthonotus were analyzed. Pandalid shrimps were active predators of macroplankton species. The diets of $P$. edwardsi and $P$. martia were quite similar and consisted primarily of benthopelagic eucarid crustaceans (Pasiphaea sp., euphausiids). P. acanthonotus is smaller, and its diet was based on smaller prey (siphonophores, hyperiids, euphausids). Seasonal changes in the diets of bathyal pandalids were important. Planktonic organisms were their main food resource, and pandalid diets corresponded with changes in the abundance of available planktonic resources in the Western Mediterranean. Thus, bathymetric distribution and abundance of pandalid shrimps along the slope were related to the influence of the mesopelagic fauna on bathyal communities, which is commonly accepted to decrease with depth. The bathymetric distribution of pandalids is also discussed in relation to a possible competitive interaction for similar resources among certain pandalid species.
\end{abstract}

\section{INTRODUCTION}

The Pandalidae (Decapoda, Caridea) comprise different genera with a high diversity of species. A large number of species within the bathyal genus Plesionika are distributed mainly in tropical and subtropical areas (Zariquiey Alvarez 1968, Crosnier \& Forest 1973). Plesionika edwardsi (Brandt, 1851), P. martia (A. Milne Edwards, 1883), and P. acanthonotus (S. I. Smith, 1882) are characteristic and abundant in bathyal mud assemblages in the deep slope of the Catalan Sea (Western Mediterranean), where environmetal parameters (temperature and salinity) in the water column are fairly constant below $200 \mathrm{~m}$ (Fredj \& Laubier 1985).

Plesionika edwardsi is distributed in the upper slope of the Catalan Sea between 256 and $512 \mathrm{~m}$, attaining its maximum abundance between 300 and $400 \mathrm{~m}$ (Abelló et al. 1988). Large sizes are linked to the submarine canyons (Cartes 1991, unpubl. data). P. martia is distributed deeper than $P$. edwardsi in the upper middle slope between 300 and $900 \mathrm{~m}$ (maximum abundance between 400 and $800 \mathrm{~m}$ ), whereas $P$. acanthonotus is found almost exclusively on the middle slope of the Catalan Sea between 300 and $1600 \mathrm{~m}$ (Abelló et al. 1988, Cartes 1991, 1993a).

The trophic habits of some pandalids have been reported on (Lagardère 1972, 1977, Wienberg 1980). Lagardère (1972) described the diet of Plesionika martia in the NW African slope. P. martia was found to prey exclusively on euphausiids (Meganyctiphanes norvegica) and mesopelagic decapods (Pasiphaea sivado). In contrast, in the Bay of Biscay this species has been suggested to scavenge (Lagardère 1977). There are no previous data about the feeding habits of the other 2 deep-Mediterranean species, although a scavenging habits has been suggested for $P$. edwardsi based on commercial catches obtained using pots (Massuti 1967, Lagardère 1976b).

Plesionika acanthonotus is a small species [Cephalothorax length (CL) between 6 and $17 \mathrm{~mm}$ ). Its bathymetric distribution overlaps with that of $P$. martia on the upper middle slope. Both species are distinctly separated by their size ranges. $P$. martia and $P$. edwardsi coincide in distribution within the submarine canyons, where a certain degree of size range overlap is also observed. 
The objective of the present paper is to describe the diets and feeding habits of the 3 deep-bathyal pandalids occurring in the western Mediterranean Sea, discussing the influence of depth and seasonality in the diet and patterns of dietary overlap among these species.

\section{MATERIAL AND METHODS}

Specimens were collected using bottom trawls on the deep slope in the Catalan Sea. CL was recorded for all specimens. A total of 83 specimens of Plesionika edwardsi, 282 of $P$. martia, and 463 of $P$. acanthonotus were studied to establish the foregut fullness $(f)$ for each species. The foregut fullness of each individual was recorded; foreguts for which $f \geq 75 \%$ were classified as very full, while values of $f<20 \%$ were classified as empty. Only contents from the fullest stomachs were selected for the analysis of diet, to reduce any bias associated with digestion (Sardà \& Valladares 1990, Cartes 1991). Consequently, only 71 specimens of $P$. edwardsi, 176 of $P$. martia, and 213 of $P$. acanthonotus were analyzed (Table 1).

Prey identification was carried out to species whenever possible. The results are presented in terms of percentage frequency of occurrence $(\% \mathrm{~F})$ and percentage abundance (\%N) (cf. Hyslop 1980, Cartes \& Sardà 1989). Percentage volume was estimated using the subjective points method (Swynnerton \& Worthington 1940) for the different prey-species or prey-groups.
General affinities between the samples (diets) were established using a multivariate cluster analysis (cf. Cartes \& Abelló 1992) for Plesionika martia and $P$. acanthonotus. Each sample combined the individuals taken in one haul. Linear correlation, applied to log-transformed data, was used as a similariy index. This index mainly considers information from the most abundant prey items. Uncommon prey items which occurred only once in the diet were removed from the data matrix. The aggregation algorithm used was UPGMA (Pielou 1984). Analysis results revealed general trends on the influence of spatial and temporal factors (depth and seasonality) in the diet of the studied species. Information obtained from previous studies on deep-water decapod diets, especially Aristeus antennatus (cf. Cartes 1991, Cartes \& Abelló 1992, Cartes 1993b), showed that dietary changes were dependent on depth and season. Based both on these results and on some general trends derived from the cluster analysis, the diet of each species was studied by seasonal period and by depth stratum. Three depth strata were established: canyons in the upper slope (380 to $530 \mathrm{~m}$ ); upper middle slope (552 to $730 \mathrm{~m})_{i}$ and lower middle slope (861 to $1249 \mathrm{~m}$ ). Detailed information about seasonal data for each established group (winter, spring-summer, autumn) is given in Table 1.

Trophic diversity was calculated using the ShannonWiener index, $H^{\prime}$ (Shannon \& Weaver 1963, Mauchline \& Gordon 1985), which is influenced both by dominance and number of species (prey items in this study) (Edgar 1983), for each of the established bathymetric and seasonal sample groups. Intraspecific and interspecific

Table 1. Sampling data for Plesionika species in the present study. C: canyon sample

\begin{tabular}{|c|c|c|c|c|c|c|}
\hline \multirow{2}{*}{ Stn } & \multirow{2}{*}{ Date } & \multirow{2}{*}{ Location } & \multirow{2}{*}{$\begin{array}{c}\text { Depth } \\
(\mathrm{m})\end{array}$} & \multicolumn{3}{|c|}{ Number of individuals } \\
\hline & & & & P. edwardsi & P. martia & P. acanthonotus \\
\hline P7 & 23 Mar 88 & $41^{\circ} 03^{\prime} \mathrm{N}, 02^{\circ} 13^{\prime} \mathrm{E}$ & $570-585$ & - & 11 & - \\
\hline P15 & 19 Feb 89 & $41^{\circ} 08^{\prime} \mathrm{N}, 01^{\circ} 56^{\prime} \mathrm{E}$ & $380-530 \mathrm{C}$ & 10 & 11 & - \\
\hline P25 & $13 \mathrm{Jul} 89$ & $41^{\circ} 03^{\prime} \mathrm{N}, 02^{\circ} 03^{\prime} \mathrm{E}$ & $650-700$ & - & 8 & - \\
\hline $\mathrm{P} 26$ & 14 Jul 89 & $41^{\circ} 03^{\prime} \mathrm{N}, 02^{\circ} 04^{\prime} \mathrm{E}$ & $640-700$ & - & 27 & 21 \\
\hline $\mathrm{P} 27$ & 14 Jul 89 & $41^{\circ} 03^{\prime} \mathrm{N}, 02^{\circ} 03^{\prime} \mathrm{E}$ & $640-660$ & - & 30 & 15 \\
\hline P288 & 14 Jul 89 & $41^{\circ} 03^{\prime} \mathrm{N}, 02^{\circ} 03^{\prime} \mathrm{E}$ & $710-730$ & - & 31 & $\sim$ \\
\hline P29 & $14 \mathrm{Jul} 89$ & $41^{\circ} 02^{\prime} \mathrm{N}, 02^{\circ} 03^{\prime} \mathrm{E}$ & $610-640$ & - & 8 & 13 \\
\hline P33 & 20 Oct 89 & $41^{\circ} 04^{\prime} \mathrm{N}, 02^{\circ} 04^{\prime} \mathrm{E}$ & $552-644$ & - & 20 & 49 \\
\hline P34 & 20 Oct 89 & $41^{\circ} 04^{\prime} \mathrm{N}, 02^{\circ} 04^{\prime} \mathrm{E}$ & $552-644$ & - & 13 & 18 \\
\hline DN2 & 21 Oct 90 & $41^{\circ} 07^{\prime} \mathrm{N}, 02^{\circ} 02^{\prime} \mathrm{E}$ & $380-525 \mathrm{C}$ & 61 & 17 & - \\
\hline $\mathrm{B} 3 / 1.5$ & $03 \mathrm{Jul} 88$ & $40^{\circ} 49^{\prime} \mathrm{N}, 01^{\circ} 36^{\prime} \mathrm{E}$ & $1011-946$ & - & - & 12 \\
\hline B.3/16 & 043 ui 88 & $40^{\circ} 49^{\prime} \mathrm{N}, 01^{\circ} 51^{\prime} \bar{E}$ & $1249-1193$ & - & - & 9 \\
\hline $\mathrm{B} 3 / 24$ & $07 \mathrm{Jul} 88$ & $39^{\circ} 53^{\prime} \mathrm{N}, 02^{\circ} 24^{\prime} \mathrm{E}$ & $980-1019$ & - & - & 10 \\
\hline $\mathrm{B} 3 / 25$ & $07 \mathrm{Jul} 88$ & $39^{\circ} 58^{\prime} \mathrm{N}, 02^{\circ} 18^{\prime} \mathrm{E}$ & $1224-1184$ & - & - & 12 \\
\hline $\mathrm{B} 5 / 11$ & 28 Oct 88 & $40^{\circ} 53^{\prime} \mathrm{N}, 01^{\circ} 45^{\prime} \mathrm{E}$ & $1036-994$ & - & - & 12 \\
\hline B5/14 & 28 Oct 88 & $40^{\circ} 56^{\prime} \mathrm{N}, 02^{\circ} 07^{\prime} \mathrm{E}$ & $1158-1034$ & - & - & 9 \\
\hline $\mathrm{B} 5 / 17$ & 29 Oct 88 & $41^{\circ} 00^{\prime} \mathrm{N}, 02^{\circ} 27^{\prime} \mathrm{E}$ & $861-1100$ & - & - & 24 \\
\hline $\mathrm{SP} / 2$ & 16 Oct 89 & $41^{\circ} 00^{\prime} \mathrm{N}, 01^{\circ} 34^{\prime} \mathrm{E}$ & $862-989$ & - & - & 5 \\
\hline
\end{tabular}


dietary overlaps were calculated based on $\% \mathrm{~N}$ results using the percentage of similarity index (Schoener 1970). Significance of differences between diets was tested using the chi-square method for contingency tables (Zar 1984) and the Spearman correlation coefficient (Sokal \& Rohlf 1979).

\section{RESULTS}

Size distribution for each species in the 3 bathymetric slope regions where pandalids were distributed is presented in Fig. 1. Although all size frequencies were significantly different between species $(p<0.01$; nonparametric Kolmogorov-Smirnov test) the highest degree of size overlap was observed between Plesionika martia and $P$. edwardsi inside submarine canyons.

\section{Diet composition}

\section{Plesionika edwardsi (canyons)}

In winter the most important prey for this species were euphausiids (Meganyctiphanes norvegica), the mesopelagic decapod Pasiphaea sivado, and polychaetes (Table 2). In contrast, fish remains were a secondary food item. Results in terms of percentage volume (Fig. 2) were similar among the various prey.

\section{No. of individuals}

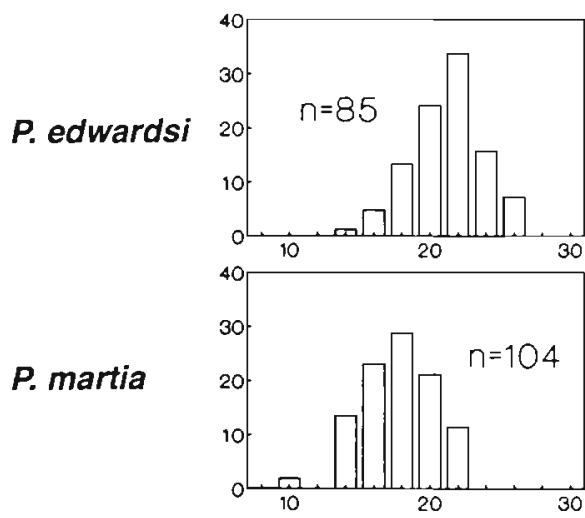

P. acanthonotus
In autumn (Table 2, Fig. 2) the dominant prey species was Pasiphaea sivado. Other important prey included polychaetes and the crustaceans Sergestes arcticus and Boreomysis arctica. The remaining prey occurred only occasionally, except that fish and shark remains were relatively common.

\section{Plesionika martia}

Cluster analysis of the diet composition for Plesionika martia showed segregation of some groups of samples (Fig. 3; see also Table 1). The most homogeneous group included all the samples collected during the springsummer period in the upper middle slope. Samples collected in autumn on the upper middle slope (Stns P33 \& P34) were grouped with a sample from a shallower submarine canyon station sampled in winter (Stn P15). The individuals collected in the canyons during autumn (Stn DN2) were clearly distinct.

Collections of Plesionika martia were grouped into 4 bathymetric and seasonal groups, only partially corresponding to groups resulting from the cluster analysis (see 'Material and methods'). The first group included a few specimens collected in a winter canyon sample $(380$ to $540 \mathrm{~m}$ ). Predominant prey in the diet (Table 3, Fig. 4) were Meganyctiphanes norvegica and Pasiphaea sivado. In this case the high incidence of euphausiids was the main factor contributing to the high affinity recorded between the second and third groups of $P$. martia. P. sivado was the dominant prey species in the diet of the specimens collected in the canyons during autumn (Stn DN2). Fish remains, polychaetes (Glycera sp., Nephthys sp., Nereidae), and Sergestes arcticus were secondary prey (Table 3 , Fig. 4).

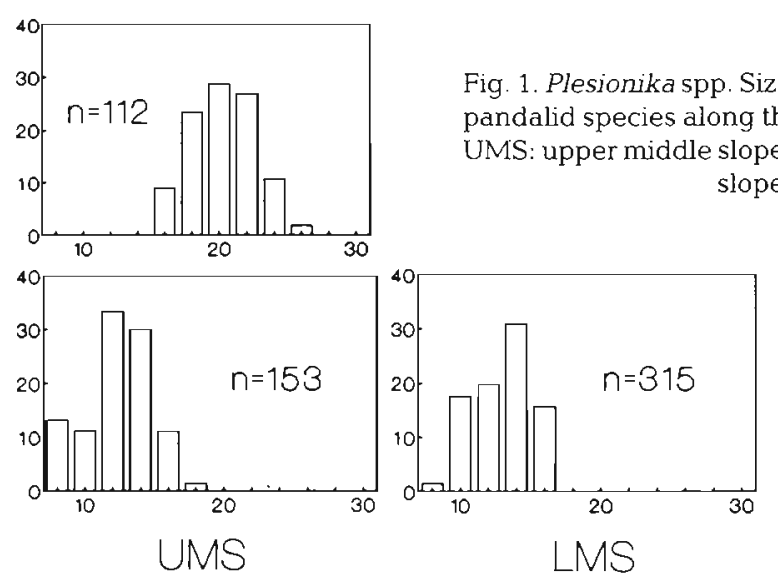


Table 2. Composition of the diet of Plesionika edwardsi in the 2 seasonal groups established. $\% \mathrm{~F}=$ frequency of occurrence; $\% \mathrm{~N}=$ percentage abundance

\begin{tabular}{|c|c|c|c|c|}
\hline \multirow{3}{*}{ No. of specimens: } & \multicolumn{4}{|c|}{ Canyons } \\
\hline & \multicolumn{2}{|c|}{$\begin{array}{c}\text { Winter (Feb) } \\
10\end{array}$} & \multicolumn{2}{|c|}{$\underset{61}{\text { Autumn }}(\mathrm{Oct})$} \\
\hline & $\% \mathrm{~F}$ & $\% \mathrm{~N}$ & $\% \mathrm{~F}$ & $\% N$ \\
\hline POLYCHAETA & 50.0 & 13.1 & 37.7 & 16.9 \\
\hline Aphroditida & 10.0 & 2.6 & - & - \\
\hline Glycera sp. & - & - & 11.5 & 4.7 \\
\hline Hyalinoecia sp. & - & - & 1.6 & 0.7 \\
\hline Eunicida (Marphysa belli) & 30.0 & 7.9 & - & - \\
\hline Nereidae & - & - & 18.0 & 7.4 \\
\hline Unidentified & 10.0 & 2.6 & 9.8 & 4.1 \\
\hline \multicolumn{5}{|l|}{ CRUSTACEA } \\
\hline \multicolumn{5}{|l|}{ Decapoda } \\
\hline \multicolumn{5}{|l|}{ Natantia } \\
\hline Mesopelagic species & 50.0 & 13.2 & 82.0 & 39.2 \\
\hline Pasiphaea sp. & 50.0 & 13.2 & 73.8 & 31.1 \\
\hline Sergestidae & - & - & 18.0 & 7.4 \\
\hline Gennadas elegans & - & - & 1.6 & 0.7 \\
\hline Unidentified Natantia & 20.0 & 5.3 & 4.9 & 2.0 \\
\hline \multicolumn{5}{|l|}{ Anomura } \\
\hline Pagurus alatus & 10.0 & 2.6 & - & - \\
\hline Euphausiacea & 60.0 & 23.7 & - & - \\
\hline Mysidacea & 10.0 & 2.6 & 21.3 & 20.3 \\
\hline Boreomysis arctica & - & - & 21.3 & 20.3 \\
\hline Unidentified & 10.0 & 2.6 & - & - \\
\hline \multicolumn{5}{|l|}{ Amphipoda } \\
\hline Gammaridea & 20.0 & 5.3 & - & - \\
\hline \multicolumn{5}{|l|}{ Isopoda } \\
\hline Cirolana borealis & 10.0 & 2.6 & - & - \\
\hline Cumacea & 10.0 & 2.6 & - & - \\
\hline Unidentified Crustacea & 20.0 & 5.3 & 1.6 & 0.7 \\
\hline \multicolumn{5}{|l|}{ MOLLUSCA } \\
\hline Bivalvia & 20.0 & 5.3 & - & - \\
\hline Abra longicallus & 10.0 & 2.6 & - & - \\
\hline Other Bivalvia & 10.0 & 2.6 & - & - \\
\hline Gastropoda & 10.0 & 2.6 & 6.6 & 3.4 \\
\hline Pteropoda & 10.0 & 2.6 & 1.6 & 0.7 \\
\hline Other Gastropoda & - & - & 6.6 & 2.7 \\
\hline Cephalopoda & - & - & 6.6 & 2.7 \\
\hline Unidentified & 20.0 & 5.3 & - & - \\
\hline \multicolumn{5}{|l|}{ ECHINODERMATA } \\
\hline Echinoidea & 20.0 & 5.3 & 1.6 & 0.7 \\
\hline CHAETOGNATA & - & - & 1.6 & 0.7 \\
\hline OSTEICHTHYES & 200 & 5.3 & 21.3 & 8.8 \\
\hline Cyclothone sp. & - & - & 3.3 & 1.4 \\
\hline Myctophidae & - & - & 6.6 & 2.7 \\
\hline Other Osteichthyes & 20.0 & 5.3 & 11.5 & 4.7 \\
\hline CHONDRICTHYES & - & - & 8.2 & 3.4 \\
\hline UNIDENTIFIED & - & - & 3.3 & 1.4 \\
\hline FORAMINIFERA & 30.0 & & 4.9 & \\
\hline Pyrgosp. & - & & 1.6 & \\
\hline Other benthic species & 30.0 & & 4.9 & \\
\hline
\end{tabular}

A fourth group included specimens collected in the spring-summer period (March to July) on the upper middle slope. Pasiphaea multidentata was the dominant prey, accounting for more than $1 / 3$ of the diet (Table 3 ). Less important were Calocaris macandreae or fish remains. Euphausiids were almost absent from the diet. The volumetric results were similar (Fig. 4). Euphausiids and fish remains were the most important prey items in the diet of the specimens collected during autumn on the upper middle slope. Gastropods (mainly pteropods) and mesopelagic decapods ( $P$. multidentata, $S$. arcticus) were secondary prey (Table 3, Fig. 4).

\section{Plesionika acanthonotus}

Two main groups were detected in the cluster analysis (Fig. 5). Temporal variations (seasonality) seemed to be more important than depth in sample aggregation. Thus, the first group included all the samples collected in summer, between 610 and $1249 \mathrm{~m}$. The second group associated all the specimens collected in autumn, between 552 and $1158 \mathrm{~m}$. Within these major groups samples seemed to be clustered on the basis of depth. Thus, as with the results for Plesionika martia, samples were classified into 4 bathymetric and seasonal groups.

Fish remains were dominant in the diet of this species on the upper middle slope in summer. Siphonophores (Chelophyes appendiculata), Pasiphaea multidentata, and isopods were secondary prey (Table 4 , Fig. 6). In contrast, some prey such as Calocaris macandreae and Meganyctiphanes norvegica were very scarce in the diet. During autumn on the upper middle slope (552 to $644 \mathrm{~m}$ ) euphausiids were more abundant than fish remains in the diet. Gastropods and hyperiids were also relatively important prey (Table 4, Fig. 6).

The diet was more diversified (Table 4 , Fig. 6) on the lower middle slope (980 to $1265 \mathrm{~m}$ ) in summer. Siphonophores and hyperiids (mainly Vibilia armata) were important prey and fish remains were also a dominant resource. Among hyperiid amphipods $V$. armata was the preferred species in the diet of Plesionika acanthono- 


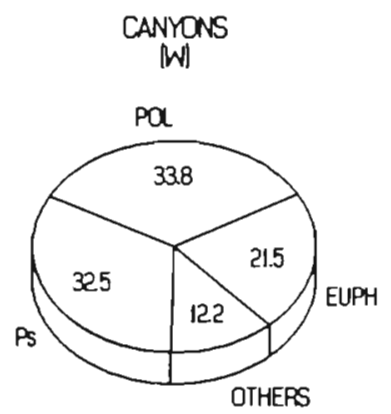

CANYONS

(A)

Fig. 2. Proportion (\% volume) of prey species in the diet of Plesionika edwardsi. POL: polychaetes; Ps: Pasiphaea sivado; Sa: Sergestes arcticus; EUPH: Euphausiacea; Bor: Boreomysis arctica; OST: Osteichthyes. (W) winter; (A) autumn

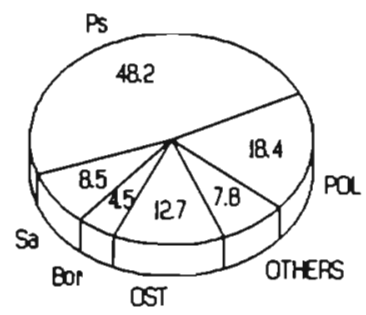

tus, probably due to its smaller size. On the lower middle slope (862 to $1165 \mathrm{~m}$ ) in autumn fish remains were predominant in the stomach contents. Euphausiids, pteropod debris, the gastropod Benthonella tenella, polychaetes, and foraminiferans were also important resources (Table 4, Fig. 6).

\section{Feeding activity}

The global results of the percentage of empty stomachs were $28 \%$ for Plesionika edwardsi, $42 \%$ for $P$. martia and $57 \%$ for $P$. acanthonotus. In $P$. martia the highest percentage of very full stomachs was observed in submarine canyons during autumn (Table 5). Outside the canyons the proportion of empty stomachs was always higher. In P. acanthonotus stomach fullness was lower when depth increased (Table 5). As a whole, considering only bathymetric results for the 3 combined Plesionika species, significant differences ( $p<0.01$; chi-square criterion) were recorded in the percentage of empty foreguts $(f<20 \%)$ within the 3 bathymetric groups established (see Fig. 1, Table 5). Thus, stomach fullness for pandalid species decreased significantly with depth.

\section{Trophic diversity}

Trophic diversity in Plesionika edwardsi was relatively low (Fig. 7). In $P$, acanthonotus diversity was relatively high for the different groups established (Fig. 7) and $H^{\prime}$ was higher in summer than in autumn for both bathymetric groups. Considering all the species as a whole within the 3 bathymetric levels studied (see 'Material and methods'), a non-parametric KruskalWallis test was applied to $H^{\prime}$ values. Only a weak significant trend $(p<0.1)$ of increasing diet diversification with increasing depth (Fig. 7) was recorded, although the obtained test value (5.61) is close to the chi-square 0.05 significance level $(5.99,2$ degrees of freedom). Comparing $H^{\prime}$ values obtained outside and inside the canyons a significant value $(p<0.05)$ was recorded, confirming a higher dietary specialization for the studied species inside the submarine canyons.

\section{Dietary affinity}

The diet overlap value was very low between the 2 seasonal groups in Plesionika edwardsi (24.573). In $P$. martia the overlap results were generally low (Table 6). The maximum value occurred when the canyon samples in autumn and the upper middle slope samples in spring-summer were compared. In contrast, the lowest overlap value occurred when the diets of these 2 bathymetric zones in autumn were compared. In $P$. acanthonotus dietary overlap values (Table 6) were quite high. The maximum value was recorded between both summer groups (60.144) whereas the lowest values occurred between the spring-summer (lower middle slope) and autumn (upper middle slope) samples.

Interspecific overlap between Plesionika edwardsi and $P$. martia samples was high in the canyons. The dietary affinity between $P$. acanthonotus and $P$. martia in the upper middle slope was relatively high only during autumn (Table 6).

Fig. 3. Aggregation of foregut content samples for Plesionika martia (linear correlation) UPGMA). UMS: upper middle slope grouping

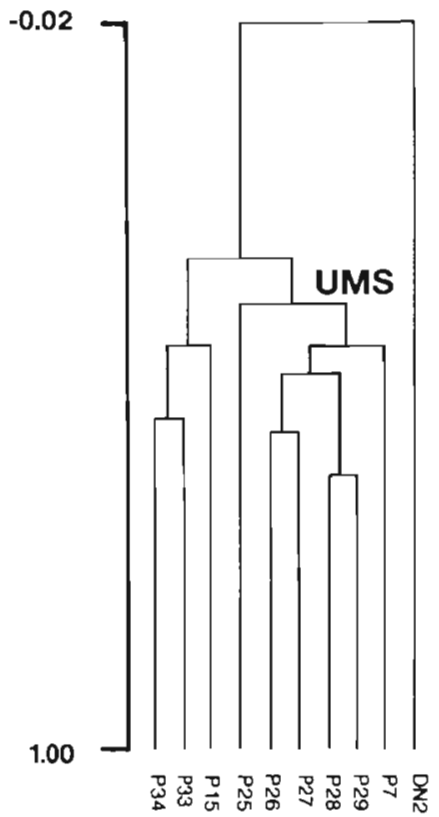


Table 3. Composition of the diet of Plesionika martia in each bathymetric and seasonal group established. \% F $=$ frequency of occurrence; $\% \mathrm{~N}=$ percentage abundance

\begin{tabular}{|c|c|c|c|c|c|c|c|c|}
\hline \multirow{4}{*}{ No. of specimens: } & \multicolumn{4}{|c|}{ Canyons $(400-500 \mathrm{~m})$} & \multicolumn{4}{|c|}{ Upper middle slope $(550-750 \mathrm{~mm})$} \\
\hline & \multirow{2}{*}{\multicolumn{2}{|c|}{$\begin{array}{c}\text { Winter }(\mathrm{Feb}) \\
10\end{array}$}} & \multirow{2}{*}{\multicolumn{2}{|c|}{$\begin{array}{l}\text { Autumn (Oct) } \\
61\end{array}$}} & \multirow{2}{*}{\multicolumn{2}{|c|}{$\begin{array}{c}\text { Spring-summer } \\
10\end{array}$}} & \multirow{2}{*}{\multicolumn{2}{|c|}{$\underset{61}{\text { Autumn (Oct) }}$}} \\
\hline & & & & & & & & \\
\hline & $\% \mathrm{~F}$ & $\% \mathrm{~N}$ & $\% \mathrm{~F}$ & $\% \mathrm{~N}$ & $\% \mathrm{~F}$ & $\% \mathrm{~N}$ & $\% \mathrm{~F}$ & $\% \mathrm{~N}$ \\
\hline HYDROZOA & - & - & - & - & 1.7 & 1.1 & 3.0 & 1.2 \\
\hline Chelophyes appendiculata & - & - & - & - & 1.7 & 1.1 & 3.0 & 1.2 \\
\hline POLYCHAETA & - & - & 41.2 & 21.9 & 6.1 & 3.4 & 6.1 & 2.4 \\
\hline Aphroditida & - & - & - & - & 3.5 & 2.3 & 6.1 & 2.4 \\
\hline Glycera sp. & - & - & 23.5 & 9.8 & - & - & - & - \\
\hline Nephthys sp. & - & - & 5.9 & 2.4 & - & - & - & - \\
\hline Nereidae & - & - & 5.9 & 2.4 & - & - & - & - \\
\hline Unidentidied & - & - & 17.6 & 7.3 & 1.7 & 1.1 & - & - \\
\hline \multicolumn{9}{|l|}{ CRUSTACEA } \\
\hline \multicolumn{9}{|l|}{ Decapoda } \\
\hline \multicolumn{9}{|l|}{ Natantia } \\
\hline Bathypelagic species & 63.6 & 38.9 & 70.6 & 41.5 & 60.9 & 38.1 & 36.4 & 14.1 \\
\hline Pasiphaea sp. & 63.6 & 38.9 & 58.8 & 31.7 & 60.0 & 34.7 & 27.3 & 10.6 \\
\hline Sergestes arcticus & - & - & 29.4 & 9.8 & 7.0 & 3.4 & 9.1 & 3.5 \\
\hline Benthic species & - & - & 5.9 & 4.8 & 4.3 & 2.9 & 6.1 & 2.4 \\
\hline Aristeus antennatus & - & - & - & - & 0.9 & 0.6 & - & - \\
\hline Plesionika sp. & - & - & - & - & 0.9 & 0.6 & 6.1 & 2.4 \\
\hline Processa canaliculata & - & - & 5.9 & 2.4 & - & - & - & - \\
\hline Alpheus glaber & - & - & - & - & 2.6 & 1.7 & - & - \\
\hline Unidentified & - & - & 5.9 & 2.4 & 10.4 & 6.3 & 12.1 & 4.7 \\
\hline \multicolumn{9}{|l|}{ Macrura } \\
\hline Calocaris macandreae & - & - & - & - & 31.3 & 19.9 & 18.2 & 7.1 \\
\hline Anomura & - & - & - & - & 3.5 & 2.2 & - & - \\
\hline Pagurus alatus & - & - & - & - & 1.7 & 1.1 & - & - \\
\hline Munida tenuimana & - & - & - & - & 1.7 & 1.1 & - & - \\
\hline Brachyura & - & - & - & - & 0.9 & 0.6 & - & - \\
\hline Post-larvae & - & - & 11.8 & 4.9 & - & - & - & - \\
\hline Unidentified & - & - & 5.9 & 2.4 & 2.6 & 1.7 & 3.0 & 1.2 \\
\hline Euphausiacea (Meganyctiphanes norvegica) & 63.9 & 38.9 & - & - & 0.9 & 0.6 & 42.4 & 17.7 \\
\hline Mysidacea & - & - & 5.9 & 4.9 & 0.9 & 0.6 & - & - \\
\hline Boreomysis arctica & - & - & 5.9 & 4.9 & - & - & - & - \\
\hline Unidentified & - & - & - & - & 0.9 & 0.6 & - & - \\
\hline \multicolumn{9}{|l|}{ Amphipoda } \\
\hline Gammaridea & - & - & - & - & 0.9 & 0.6 & 3.0 & 1.2 \\
\hline Lysianassidae & - & - & - & - & 0.9 & 0.6 & - & - \\
\hline Synopiidae & - & - & - & - & - & - & 3.0 & 1.2 \\
\hline Hyperiidea & 9.1 & 5.6 & - & - & 5.2 & 3.3 & 15.2 & 6.0 \\
\hline Phrosina sp. & 9.1 & 5.6 & - & - & 3.5 & 2.2 & 6.1 & 2.4 \\
\hline Vibilia armata & - & - & - & - & - & - & 3.0 & 1.2 \\
\hline Other Hyperiidea & - & - & - & - & 1.7 & 1.1 & 6.1 & 2.4 \\
\hline Isopoda (Cirolana borealis) & - & - & - & - & 4.3 & 2.8 & - & - \\
\hline Tanaidacea Apseudomorpha & - & - & - & - & 2.6 & 1.1 & - & - \\
\hline Cumacea (Epileucon longirostris) & - & - & - & - & 1.7 & 1.1 & - & - \\
\hline Ostracoda Cypridinidae & - & - & - & - & 0.9 & 0.6 & - & - \\
\hline Copepoda Harpacticoidea & - & - & - & - & - & - & 3.0 & 1.2 \\
\hline Unidentified Crustacea & 9.1 & 5.6 & - & - & 1.7 & 1.1 & 6.1 & 2.4 \\
\hline MOLLUSCA & & & & & & & & \\
\hline Bivalvia & - & - & - & - & 1.7 & 1.1 & - & - \\
\hline Gastropoda & - & - & 17.6 & 7.3 & 2.6 & 1.7 & 27.3 & 11.8 \\
\hline Pteropoda & - & - & - & - & 2.6 & 1.7 & 18.2 & 7.1 \\
\hline Benthonella tenera & - & - & - & - & - & - & 12.1 & 4.7 \\
\hline Other Gastropoda & - & - & 17.6 & 7.3 & - & - & - & - \\
\hline Unidentified & - & - & - & - & - & - & 6.1 & 2.4 \\
\hline ECHINODERMATA & - & - & - & - & 1.7 & 1.2 & 6.1 & 2.4 \\
\hline Echinoidea & - & - & - & - & 0.9 & 0.6 & 3.0 & 1.2 \\
\hline Ophiuroidea & - & - & - & - & 0.9 & 0.6 & 3.0 & 1.2 \\
\hline CHAETOGNATA & 9.1 & 5.6 & 17.6 & 7.3 & 13.0 & 8.5 & 42.4 & 38.1 \\
\hline OSTEICHTHYES & 9.1 & 5.6 & 17.6 & 7.3 & 13.0 & 8.5 & 42.4 & 38.1 \\
\hline Cyclothone sp. & - & - & - & - & - & - & 3.0 & 2.4 \\
\hline Myctophidae & - & - & 5.9 & 2.4 & 2.6 & 1.7 & 6.1 & 3.6 \\
\hline Other Osteich thyes & 9.1 & 5.6 & 11.8 & 4.9 & 13.0 & 6.8 & 33.3 & 32.1 \\
\hline
\end{tabular}


Table 3 (continued)

\begin{tabular}{|c|c|c|c|c|c|c|c|c|}
\hline \multirow{3}{*}{ No. of specimens: } & \multicolumn{4}{|c|}{ Canyons $(400-500 \mathrm{~m})$} & \multicolumn{4}{|c|}{ Upper middle slope (550-750 $\mathrm{mm}$ ) } \\
\hline & \multicolumn{2}{|c|}{$\begin{array}{c}\text { Winter (Feb) } \\
10\end{array}$} & \multicolumn{2}{|c|}{$\underset{61}{\text { Autumn }}(\mathrm{Oct})$} & \multicolumn{2}{|c|}{$\begin{array}{c}\text { Spring-summer } \\
10\end{array}$} & \multicolumn{2}{|c|}{$\begin{array}{l}\text { Autumn (Oct) } \\
61\end{array}$} \\
\hline & $\% F$ & $\% \mathrm{~N}$ & $\% \mathrm{~F}$ & $\% \mathrm{~N}$ & $\% F$ & $\% \mathrm{~N}$ & $\% F$ & $\% \mathrm{~N}$ \\
\hline OTHERS & - & - & - & - & 0.9 & 0.6 & - & - \\
\hline UNIDENTIFIED & - & - & 5.9 & 2.4 & 0.9 & 0.6 & 12.1 & 4.7 \\
\hline FORAMINIFERA & - & & 11.8 & & 4.3 & & 12.1 & \\
\hline Uvigerina sp. & - & & - & & 1.7 & & - & \\
\hline Ammodiscus sp. & - & & - & & 0.9 & & - & \\
\hline Gyroidina soldanii & - & & 5.9 & & 3.5 & & 6.1 & \\
\hline Globigerinae & - & & 5.9 & & 4.3 & & 6.1 & \\
\hline Mud & - & & - & & - & & 3.0 & \\
\hline Micromolluscs remains & - & & - & & 1.7 & & 9.1 & \\
\hline
\end{tabular}
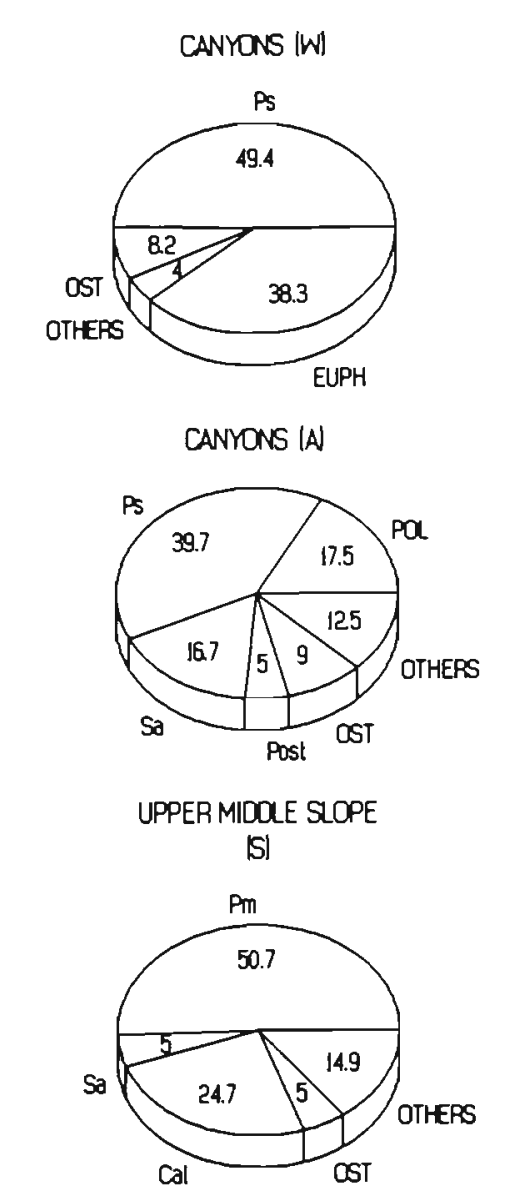

Fig. 4. Proportion (\% volume) of prey species in the diet of Plesionika martia. POL: polychaetes; Ps: Pasiphaea sivado; Pm: Pasiphaea multidentata; Sa: Sergestes arcticus; NAT: natantian; Cal: Calocaris macandreae; Post: decapod postlarvae; EUPH: Euphausiacea; OST: Osteichthyes. W: winter; A: autumn; S: summer

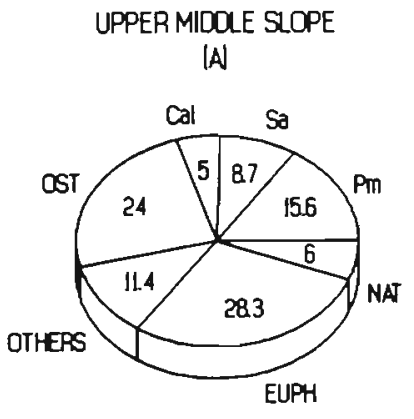

Dietary affinities among all the bathymetric and seasonal groups established were tested using the chisquare criterion for contingency tables (Zar 1984). No comparisons were statistically significant $(p<0.05)$. So, the dietary overlap results in this study must be considered as relative. Results of the semi-quantitative Spearman coefficient (Table 6), whes e the order of importance by prey is the factor determining dietary affinities, were only significant $(\mathrm{p}<0.05)$ between the 2 Plesionika species occurring inside submarine canyons during autumn and between both seasonal groups of Plesionika acanthonotus established on the lower middle slope.

\section{DISCUSSION}

Pandalid shrimps on the deep slope in the Catalan Sea were active predators on macroplankton, although

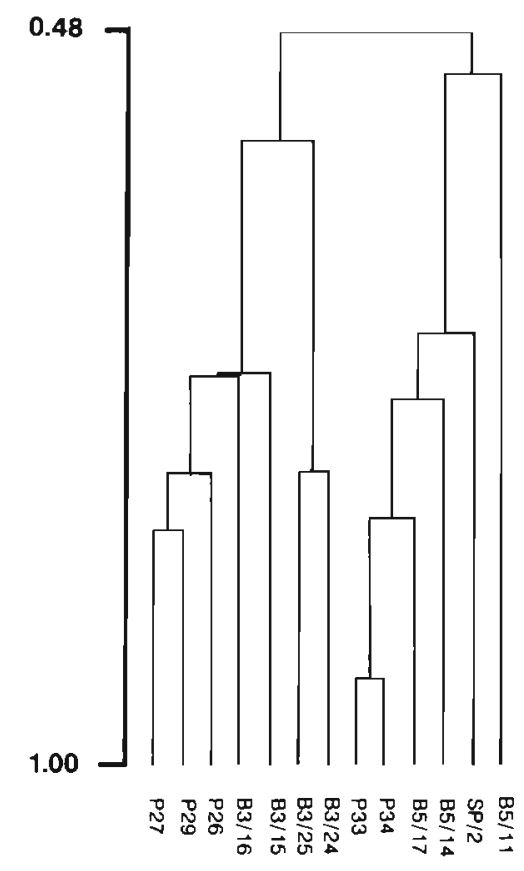

Fig. 5. Aggregation of foregut content samples for Plesionika acanthonotus (linear correlation / UPGMA]

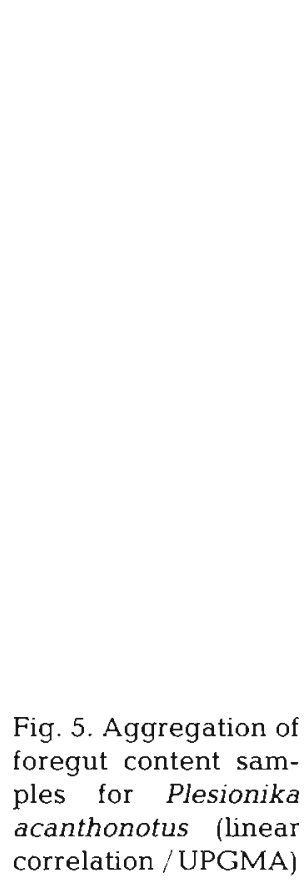


Table 4. Composition of the diet of Plesionika acanthonotus in each bathymetric and seasonal group established. \%F $=$ frequency of occurrence $; \% \mathrm{~N}=$ abundance

\begin{tabular}{|c|c|c|c|c|c|c|c|c|}
\hline \multirow{3}{*}{ No. of specimens: } & \multicolumn{4}{|c|}{ Upper middle slope } & \multicolumn{4}{|c|}{ Lower middle slope } \\
\hline & \multicolumn{2}{|c|}{$\begin{array}{c}\text { Summer (Jul) } \\
49\end{array}$} & \multicolumn{2}{|c|}{$\begin{array}{l}\text { Autumn (Oct) } \\
71\end{array}$} & \multicolumn{2}{|c|}{$\underset{47}{\text { Summer (Jul) }}$} & \multicolumn{2}{|c|}{$\underset{50}{\text { Autumn }}(O c t)$} \\
\hline & $\% \mathrm{~F}$ & $\% N$ & $\% \mathrm{~F}$ & $\% \mathrm{~N}$ & $\% \mathrm{~F}$ & $\% \mathrm{~N}$ & $\% \mathrm{~F}$ & $\% \mathrm{~N}$ \\
\hline HYDROZOA & 18.4 & 10.9 & 5.6 & 1.8 & 38.3 & 13.0 & 10.0 & 5.2 \\
\hline Chelophyes appendiculata & - & - & - & - & 1.7 & 1.1 & 3.0 & 1.2 \\
\hline POLYCHAETA & 8.2 & 4.4 & 9.7 & 4.8 & 21.3 & 6.6 & 22.0 & 7.9 \\
\hline Aphroditida & - & - & 4.2 & 1.8 & 6.4 & 2.2 & - & - \\
\hline Glycera sp. & - & - & - & - & - & - & 2.0 & 0.9 \\
\hline Hyalinoecia sp. & - & - & - & - & - & - & 2.0 & 0.9 \\
\hline Nereidae & - & - & - & - & - & - & 2.0 & 0.9 \\
\hline Spionidae & - & - & - & - & 8.5 & 2.2 & 8.0 & 3.5 \\
\hline Other (Serpulidae) & - & - & - & - & 2.1 & 0.7 & - & - \\
\hline Unidentidied & 8.2 & 4.4 & 8.3 & 3.0 & 4.3 & 1.5 & 4.0 & 1.8 \\
\hline \multicolumn{9}{|l|}{ CRUSTACEA } \\
\hline \multicolumn{9}{|l|}{ Decapoda } \\
\hline \multicolumn{9}{|l|}{ Natantia } \\
\hline Bathypelagic species & 10.2 & 3.3 & 4.2 & 1.8 & 19.1 & 6.6 & 24.0 & 10.3 \\
\hline Sergestidae & - & - & 1.4 & 0.6 & - & - & - & - \\
\hline Acanthephyra sp. & - & - & - & - & 6.4 & 2.2 & 4.0 & 1.8 \\
\hline Pasiphaea multidentata & 10.2 & 3.3 & 2.8 & 1.2 & 12.8 & 4.4 & 20.0 & 8.6 \\
\hline \multicolumn{9}{|l|}{ Benthic species } \\
\hline Plesionika sp. & 2.0 & 1.1 & - & - & 4.3 & 1.5 & 2.0 & 0.9 \\
\hline Unidentified & 4.1 & 2.2 & 2.8 & 1.2 & 10.6 & 3.6 & 4.0 & 1.8 \\
\hline \multicolumn{9}{|l|}{ Macrura } \\
\hline C. macandreae & 4.1 & 2.2 & 1.4 & 0.6 & 2.1 & 0.7 & - & - \\
\hline Anomura & & & & & & & & \\
\hline Pagurus alatus & 6.1 & 3.3 & 1.4 & 0.6 & - & - & - & - \\
\hline Unidentified & 6.1 & 3.3 & 1.4 & 0.6 & 6.4 & 2.2 & 6.0 & 2.6 \\
\hline Euphausiacea & 2.0 & 1.1 & 68.1 & 32.0 & 6.4 & 2.9 & 30.0 & 15.5 \\
\hline Mysidacea & - & - & - & - & 8.5 & 2.9 & - & - \\
\hline Boreomysis arctica & - & - & - & - & 6.4 & 2.2 & - & - \\
\hline Unidentified & - & - & - & - & 2.1 & 0.7 & - & - \\
\hline Amphipoda & & & & & & & & \\
\hline Gammaridea & 4.1 & 2.2 & 2.8 & 1.2 & 4.3 & 1.5 & 6.0 & 2.6 \\
\hline Lysianassidae & - & - & - & - & 2.1 & 0.7 & - & - \\
\hline Oedicerotidae & - & - & 1.4 & 0.6 & - & - & - & - \\
\hline Rhachotropis sp. & 4.1 & 2.2 & - & - & - & - & - & - \\
\hline Synopiidae & - & - & 1.4 & 0.6 & - & - & - & - \\
\hline Unidentified & - & - & - & - & 2.1 & 0.7 & 6.0 & 2.6 \\
\hline Hyperiidea & 4.1 & 6.6 & 12.5 & 5.4 & 55.3 & & 8.0 & 4.4 \\
\hline Phrosina semilunata & 6.1 & 3.3 & 4.2 & 1.8 & 19.1 & 6.5 & 2.0 & 0.9 \\
\hline Vibilia armata & - & - & 5.6 & 2.4 & 31.9 & 12.3 & 2.0 & 0.9 \\
\hline Phronima sedentaria & 6.1 & 3.3 & 2.8 & 1.2 & 8.5 & 3.6 & 6.0 & 2.6 \\
\hline Isopoda & 16.3 & 10.1 & 4.2 & 1.8 & 2.1 & 2.2 & - & - \\
\hline Cirolana borealis & 6.1 & 3.3 & - & - & 2.1 & 0.7 & - & - \\
\hline Munnopsurus atlanticus & 8.2 & 4.4 & 4.2 & 1.8 & - & - & - & - \\
\hline Other Paraselloidea & 2.0 & 1.1 & - & - & - & - & - & - \\
\hline Unidenditified & 2.0 & 1.1 & - & - & 4.3 & 1.5 & - & - \\
\hline Tanaidacea Apseudomorpha & - & - & 4.2 & 1.8 & - & - & - & - \\
\hline Cumacea & 2.0 & 1.1 & - & - & 8.5 & 2.9 & 4.0 & 1.8 \\
\hline Epileucon longirostris & 2.0 & 1.1 & - & - & 6.4 & 2.2 & - & - \\
\hline Cyclaspis longirostris & - & - & - & - & 2.1 & 0.7 & 4.0 & 1.8 \\
\hline Unidentified & - & - & - & - & - & - & - & - \\
\hline Ostracoda & 2.0 & 1.1 & 1.4 & 0.6 & 4.3 & 1.5 & - & - \\
\hline Myodocopa & - & - & - & - & 2.1 & 0.7 & - & - \\
\hline Platycopa & 2.0 & 1.1 & 1.4 & 0.6 & 2.1 & 0.7 & - & - \\
\hline Copepoda & - & - & 1.4 & 0.6 & 4.3 & 1.5 & 4.0 & 1.8 \\
\hline Harpacticoidea & - & - & 1.4 & 0.6 & - & - & - & - \\
\hline Calanoideà & - & - & - & - & 4.3 & 1.5 & 4.0 & 1.8 \\
\hline Unidentified Crustacea & 2.0 & 1.1 & 5.6 & 2.4 & 4.3 & 1.5 & 2.0 & 0.9 \\
\hline PYCNOGONIDA & - & - & - & - & - & - & 2.0 & 0.9 \\
\hline MOLLUSCA & & & & & & & & \\
\hline Bivalvia & - & - & 2.8 & 1.2 & - & - & 4.0 & 1.8 \\
\hline Abra longicallus & - & - & 2.8 & 1.2 & - & - & 4.0 & 1.8 \\
\hline Other Bivalvia & - & - & - & - & - & - & 4.0 & 1.8 \\
\hline
\end{tabular}


Table 4 (continued)

\begin{tabular}{|c|c|c|c|c|c|c|c|c|}
\hline \multirow{3}{*}{ No. of specimens: } & \multicolumn{4}{|c|}{ Upper middle slope } & \multicolumn{4}{|c|}{ Lower middle slope } \\
\hline & \multicolumn{2}{|c|}{$\begin{array}{c}\text { Summer (Jul) } \\
49\end{array}$} & \multicolumn{2}{|c|}{$\begin{array}{c}\text { Autumn (Oct) } \\
71\end{array}$} & \multicolumn{2}{|c|}{$\underset{47}{\text { Summer (Jul) }}$} & \multicolumn{2}{|c|}{$\begin{array}{l}\text { Autumn (Oct) } \\
50\end{array}$} \\
\hline & $\% F$ & $\% \mathrm{~N}$ & $\% \mathrm{~F}$ & $\% \mathrm{~N}$ & $\% \mathrm{~F}$ & $\% \mathrm{~N}$ & $\% \mathrm{~F}$ & $\% \mathrm{~N}$ \\
\hline Gastropoda & 8.1 & 5.4 & 19.4 & 7.7 & 6.4 & 3.0 & 22.0 & 13.8 \\
\hline Pteropoda & 8.1 & 5.4 & 12.4 & 4.7 & 4.3 & 1.5 & 16.0 & 6.9 \\
\hline Alvania sp. & - & - & 1.4 & 0.6 & - & - & - & - \\
\hline Benthonella tenella & - & - & 4.2 & 1.8 & 4.3 & 1.5 & 16.0 & 6.0 \\
\hline Other Gastropoda & - & - & 1.4 & 0.6 & - & - & 2.0 & 0.9 \\
\hline Cephalopoda & - & - & - & - & - & - & 2.0 & 0.9 \\
\hline Unidentified & 2.0 & 1.1 & 9.7 & 4.1 & 2.1 & 0.7 & 2.0 & 0.9 \\
\hline ECHINODERMATA & & & & & & & & \\
\hline Ophiuroidea & - & - & 1.4 & 0.6 & - & - & - & - \\
\hline SIPUNCULOIDEA & - & - & 6.9 & 3.0 & - & - & - & - \\
\hline OSTEICHTHYES & 71.4 & 36.9 & 61.1 & 26.1 & 66.0 & 22.5 & 58.0 & 28.5 \\
\hline Myctophidae & 6.1 & 4.4 & 4.2 & 1.8 & - & - & - & - \\
\hline Other Osteichthyes & 65.3 & 32.6 & 56.9 & 24.3 & 66.0 & 22.5 & 58.0 & 28.5 \\
\hline Unidentified & - & - & 2.8 & 1.2 & 2.1 & 0.7 & 2.0 & 0.9 \\
\hline FORAMINIFERA & 34.7 & & 15.3 & & 17.0 & & 38.0 & \\
\hline Robulus sp. & 4.1 & & - & & 2.1 & & 4.0 & \\
\hline Uvigerina sp. & 2.0 & & - & & - & & 6.0 & \\
\hline Pyrgo sp. & - & & 1.4 & & 4.3 & & - & \\
\hline Other benthic species & 2.0 & & - & & 4.3 & & - & \\
\hline Globigerinae & 34.7 & & 13.9 & & 6.4 & & 34.0 & \\
\hline Mud & - & & 1.4 & & - & & 2.0 & \\
\hline Micromolluscs remains & 8.2 & & 8.3 & & - & & 8.0 & \\
\hline
\end{tabular}

they also made substantial use of benthic resources. The cycle of daily activity was also an important factor in their feeding habits (Cartes 1991, unpubl. data). Thus, the diet of Plesionika martia underwent an important quantitative change on the upper middle slope between daytime and nighttime. The daytime diet was based almost exclusively on the mesopelagic shrimp Pasiphaea multidentata.

The diet of Plesionika edwardsi consisted primarily of benthopelagic eucarid crustaceans, especially $P_{a s i-}$ phaea sivado. Plesionika martia has been described as a specialized predator of bathypelagic species (Lagardère 1972). In the present study, although the prey species consumed changed with depth, benthopelagic eucarids were the predominant prey in pandalid diets. P. edwardsi also feeds on endobenthic prey, mostly polychaetes. Such feeding behaviour is fairly unusual among the pandalids here studied, although $P$. martia exhibited a similar trend in the submarine canyons. Polychaete consumption among the pandalids was considerable on the upper slope in the Bay of Biscay (Lagardère 1977). The feeding habits of $P$. martia and $P$. edwardsi were thus similar in the submarine canyons, the only slope region where these 2 species co-occur, (also exhibiting a certain degree of size overlap). P. edwardsi occurred in the submarine canyons on the upper middle slope but was replaced by P. martia at greater depths (Abelló et al. 1988, own unpubl. data). According to the hypothesis of Rex (1977), both the high trophic level occupied by the 2 pandalids and the relatively high affinity exhibited between their diets may be consistent with a bathymetric displacement between these species.

Plesionika acanthonotus is smaller, and although it exploits a similar type of resource, it directed its activities towards smaller prey items (siphonophores, hyperiids, euphausiids). Benthic prey and Osteichthyes remains were also important. The feeding strategies of $P$. acanthonotus and P. martia are similar (directed to pelagic resources). However, selection of different prey sizes by these 2 species attenuates the exploitation of the same resource (cf. Macpherson 1981). Predation on euphausiids in autumn, the only common resource exploited, probably does not imply interspecific competition, in view of a possible different selection of prey size and because of the very high abundance of euphausiids in the plankton during autumn (Franqueville 1971). A similar resource partitioning by size has been suggested to occur among pasiphaeid species (Lagardère 1976b).

Scavenging is a secondary activity in the feeding habits of pandalids. The proportion of Osteichthyes and Chondrichthyes remains in the diet of Plesionika edwardsi and $P$. martia was small (5 to $15 \%$ ), with occasional otoliths or mandible arches of myctophids and Cyclothone sp., suggestive of active hunting of small fishes. These results conflict with the classifica- 


\section{UPPER MIDOLE SLOPE ISI}

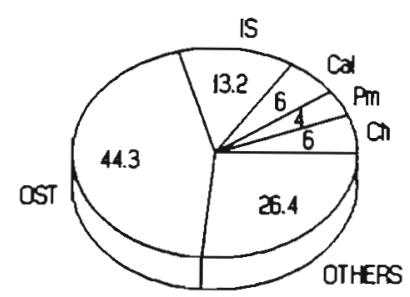

UPPER MIDOAE SLOPE (A)

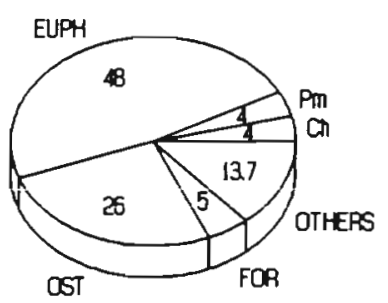

LOWER MIDQL SLOPE ISI

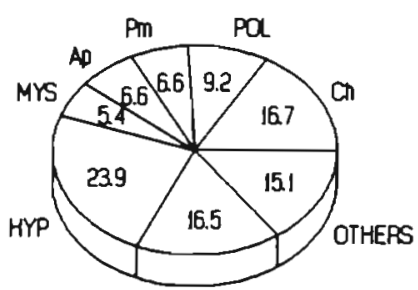

OST

\section{LOWER MIDOLE SLOPE (A)}

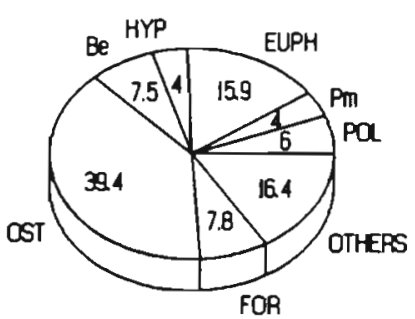

Fig. 6. Proportion (\% volume) of prey species in the diet of Plesionika acanthonotus. Ch: Siphonophora; POL: polychaetes; Ap: Acanthephyra sp.; Pm: Pasiphaea multidentata; Cal: Calocaris macandreae; EUPH: Euphausiacea; MYS: Mysidacea; HYP: Hyperiidea; IS: Isopoda; Be: Benthonella tenella; OST: Osteichthyes; FOR: Foraminifera. (A): autumn; (S): summer

tion of $P$. edwardsi as an active scavenger (Lagardère 1976a) based on large catches achieved using pots (Massuti 1967). P. acanthonotus seemed to exhibit the most pronounced scavenging activity, though in many cases $(60 \%)$ Osteichthyes remains consisted of large isolated scales. Its advanced digestion state (Cartes 1991) precluded the possibility of net feeding; rather, scales were probably ingested in the water column (Hopkins \& Baird 1975), an activity more in keeping

with passive predation on slow-moving resources above the bottom than with scavenger behaviour.

The proportion of benthic prey items in the diets of pandalids was low. Such prey items tended to be slow-moving (sedimented pteropod shells, molluscs, and fish remains), while mobile epibenthic resources such as peracarids always occurred sporadically in the diet.

Seasonal changes in pandalid diets followed a common pattern with respect to euphausiids, which were basic components of the diets of both Plesionika martia and $P$. acanthonotus on the middle slope in autumn. In the submarine canyons the seasonal pattern was different, and euphausiids were only important in winter. This same pattern of feeding on euphausids was common to all the bathyal decapods in the study area (Cartes 1991) and was in accordance with the abundance of this group in the water column (Franqueville 1971). Siphonophores and hyperiids were also seasonally important in the diet of $P$. acanthonotus, being abundant in summer. The siphonophore species identified (Chelophyes appendiculata) and certain hyperiid species (Phrosina semilunata, Phronima sedentaria) attain peak abundance levels in the Western Mediterranean in summer (Franqueville 1971). Seasonal differences in the diets of bathyal decapods in the Catalan Sea were most important for those species which, like the pandalids, relied on planktonic organisms as their main resource (Cartes 1991).

The influence of the mesopelagic fauna on bathyal benthic communities is commonly accepted to decrease with depth (Lagardère 1977). Pérès (1958) reported that euphausiid shoals tended to aggregate near topographical features in the sea bed, such as the slopes of submarine canyons down to $1000 \mathrm{~m}$. Some

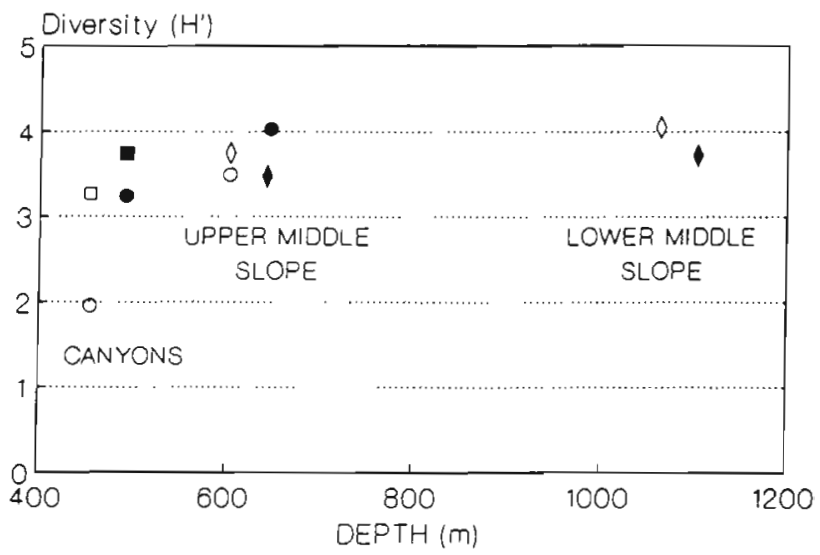

Fig. 7. Trophic diversity $\left(H^{\prime}\right)$ in the diet of pandalids determined by depth and seasonal groups. $(0$, Plesionika acanthonotus; $(\square, \square)$ P. edwardsi; $(0, \bullet)$ P. martia. Open symbols winter-summer values; filled symbols: autumn values 
Table 5. Percentage of stomach fullness (f) in Plesionika edwardsi, $P$. acanthonutus and $P$. martia by depth and season

\begin{tabular}{|c|c|c|c|c|c|c|c|}
\hline & & \multicolumn{2}{|c|}{$\begin{array}{c}\text { Canyons } \\
(400-500 \mathrm{~m})\end{array}$} & \multicolumn{2}{|c|}{$\begin{array}{l}\text { Upper middle slope } \\
\quad(550-750 \mathrm{~m})\end{array}$} & \multicolumn{2}{|c|}{$\begin{array}{l}\text { Lower middle slope } \\
(1000-1200 \mathrm{~m})\end{array}$} \\
\hline & & Feb & Oct & Mar-Jul & Oct & Jul & Oct \\
\hline P. edwardsi & $\begin{array}{l}\text { No. of specimens } \\
f<20 \% \\
20 \% \leq f<75 \% \\
f \geq 75 \%\end{array}$ & $\begin{array}{r}12 \\
41.6 \\
50.0 \\
8.3\end{array}$ & $\begin{array}{l}71 \\
25.4 \\
43.6 \\
31.0\end{array}$ & & & & \\
\hline P. martia & $\begin{array}{l}\text { No. of specimens } \\
f<20 \% \\
20 \% \leq f<75 \% \\
f \geq 75 \%\end{array}$ & $\begin{array}{r}192 \\
42.1 \\
47.4 \\
10.5\end{array}$ & $\begin{array}{l}20 \\
20.0 \\
45.0 \\
35.0\end{array}$ & $\begin{array}{l}175 \\
40.0 \\
36.0 \\
24.0\end{array}$ & $\begin{array}{l}68 \\
45.6 \\
35.3 \\
19.1\end{array}$ & & \\
\hline P. acanthonotus & $\begin{array}{l}\text { No. of specimens } \\
f<20 \% \\
20 \% \leq f<75 \% \\
f \geq 75 \%\end{array}$ & & & $\begin{array}{l}75 \\
58.6 \\
37.4 \\
4.0\end{array}$ & $\begin{array}{l}72 \\
23.6 \\
44.5 \\
31.9\end{array}$ & $\begin{array}{c}129 \\
48.1 \\
41.8 \\
10.1\end{array}$ & $\begin{array}{r}187 \\
75.9 \\
19.3 \\
4.8\end{array}$ \\
\hline
\end{tabular}

Table 6. Intraspecific and interspecific dietary overlaps between the different bathymetric and seasonal groups in Plesionika species. S; similanty; r: Spearman correlation; ("): significant value; UMS: upper middle slope; LMS: lower middle slope

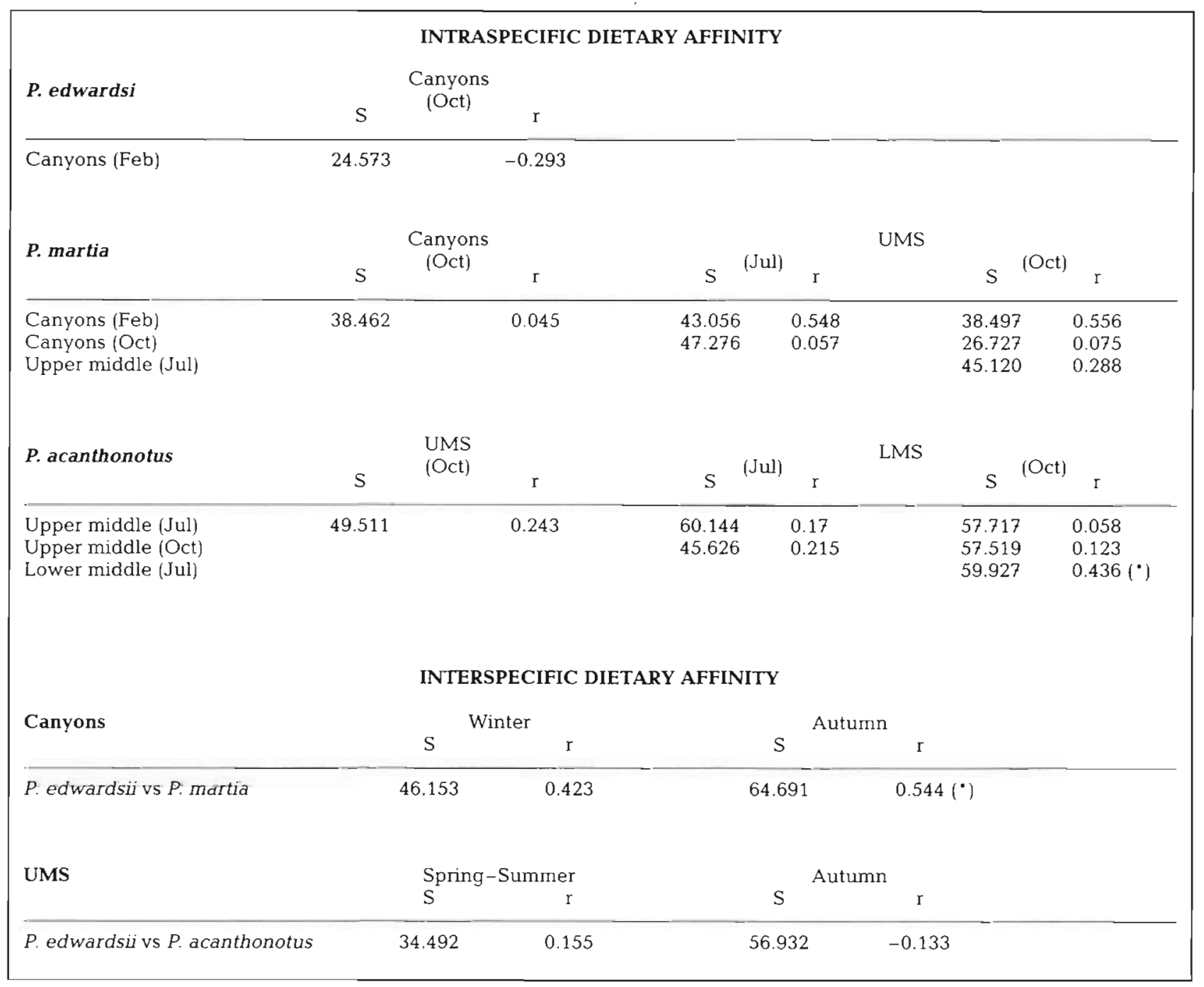


samples taken in the benthic boundary layer have pointed to a decrease in euphausiid densities on the lower slope (Hargreaves et al. 1984). In accordance with this finding, euphausiids were more abundant in the shallowest samples $(650$ to $730 \mathrm{~m})$ than on the lower middle slope (862 to $1265 \mathrm{~m}$ ) in the diet of pandalid species in the present study. In the Catalan Sea, there is a distinct boundary, at around 1200 to $1300 \mathrm{~m}$, limiting the influence of euphausiids and mesopelagic fishes in the diets of deep-water decapods (Cartes 1991). In contrast, siphonophores and hyperiids were most abundant in the deepest samples in the diet of Plesionika acanthonotus. The abundance of gelatinous plankton near the bottom is apparently higher below $1000 \mathrm{~m}$, as indicated by direct results (Hargreaves et al. 1984, Hargreaves 1985) and by indirect results, such as on the bathymetric distribution of benthopelagic fishes of the genus Alepocephalus (Gordon \& Mauchline 1990, Stefanescu 1991), which are highly specialized exploiters of this type of resource.

Dietary diversity in pandalids seemed to be associated with sample depth. Dietary diversity was low in Plesionika edwardsi as compared to the other 2 species outside the canyons. This pattern was also observed for $P$. martia in the canyons. The cause underlying this finding could be a specialization towards a specific type of resource (Pasiphaea sivado, euphausiids), which is probably more abundant in a more highly productive zone (Haedrich et al. 1980). Thus, the consistently higher stomach fullness values in the canyons were also attributable to greater food abundance there. In $P$. acanthonotus, the deepest distributed pandalid species, dietary diversity was higher as specific resources became less abundant in the environment. On the lower middle slope the behavioural pattern of $P$. acanthonotus was more like that of a non-selective predator (Dayton \& Hessler 1972).

Because of the rather specialized nature of pandalid diets, the distribution and abundance of these species seemed to be strongly conditioned by the abundance of their preferred resources. Thus, $P$. martia based more than one-third of its diet on a single prey item (Pasiphaea multidentata) in summer. Preferred prey items also included Meganyctiphanes norvegica and Calocaris macandreae. The abundance of all these species falls off sharply below $1000 \mathrm{~m}$ (Lagardère 1977 , Cartes 1991), where the distribution range of $P$. martia (depth distribution 400 to $900 \mathrm{~m}$ ) ends. The drop in abundance of $P$. acanthonotus towards 1200 to $1300 \mathrm{~m}$ may well be related to the nearly total absence of euphausiids below this level.

Despite the dependence of bathyal pandalids on planktonic resources, these species were not present in samples collected from various levels in the water column (planktonic hauls). Predatory activity by pandalids on planktonic organisms would thus appear to take place in the benthic boundary layer located a few meters above the bottom (Wishner 1980, Hargreaves 1984, Angel 1990). The results of some samples obtained over $24 \mathrm{~h}$ sampling periods carried out in the same area suggest that the periodicity of such activity was unclear and may be irregular (Cartes et al. 1993).

Acknowledgements. The present study was carried out in the framework of the BATIMAR (ref. PAC 86-008/TD 821), PONT'89 and PONT'90 research projects, which were supported by the 'CAYCIT' and 'CSIC'. The author thanks all the participants, Ms G. Fuster and Mr J. M. Anguita for their technical assistance and Mr R. Sacks for the English translation.

\section{LITERATURE CITED}

Abelló, P., Valladares, F. J., Castellón, A. (1988). Analysis of the structure of decapod crustacean assemblages off the Catalan coast (North-West Mediterranean). Mar. Biol. 98: 39-49

Angel, M. V. (1990). Life in the benthic boundary layer: connections to the mid-water and sea floor. Phil. Trans. R. Soc. Lond. 331: 15-28

Cartes, J. E. (1991). Análisis de las comunidades y estructura trófica de los crustáceos decápodos batiales del Mar Catalán Tesis doctoral, Universidad Politécnica de Catalunya

Cartes, J. E. (1993a). Deep-sea decapod fauna of the Western Mediterranean: bathymetric distribution and biogeographic aspects. Crustaceana (in press)

Cartes, J. E. (1993b). Feeding habits of pasiphaeid shrimps close to the bottom in the Western Mediterranean slope. Mar. Biol. (n press)

Cartes, J. E., Abelló, P. (1992). Comparative feeding habits of polychelid lobsters in the western Mediterranean. Mar. Ecol. Prog. Ser. 84: 139-150

Cartes, J. E., Sardà, F. (1989). Feeding ecology of the deepwater aristeid crustacean Aristeus antennatus. Mar. Ecol. Prog. Ser. 54: 229-238

Cartes, J. E., Sardà, F., Compány, J. B., Lleonart, J. (1993). Day-night migrations by deep-sea decapod crustaceans in experimental samplings in the Western Mediterranean Sea. J. exp. mar. Biol. Ecol. (in press)

Crosnier, A., Forest, J. (1973). Les crevettes profondes de l'Atlantique oriental tropical. Faune trop. 19: 1-409

Dayton, P. K., Hessler, R. R. (1972). Role of biological disturbace in maintaining diversity in the deep sea. Deep Sea Res. 19: 199-208

Edgar, G. J., (1983). The ecology of south-east Tasmanian phytal animal communities. III. Patterns of species diversification. J. exp. mar. Biol. Ecol. 70: 181-203

Franqueville, C. (1971). Macroplancton profond (Invertébrés) de la Méditerranée nord-occidentale. Tethys 3(1): 11-56

Fredj, G., Laubier, L. (1985). The deep Mediterranean benthos. In: Moraitou-Apostolopoulou, M., Kiortsis, V. (eds.) Mediterranean marine ecosystems. Plenum Press, New York, p. 109-146

Gordon, J. D. M., Mauchline, J. (1990). Depth-related trends in diet of a deep-sea bottom-living fish assemblage of the Rockall Trough. In: Barnes, M., Gibson, R. N. (eds.) Trophic relationships in the marine environment. Proc. 24th Eur. Mar. Biol. Symp. Aberdeen University Press, 
Aberdeen, p. 439-452

Haedrich, R. L., Rowe, G. T., Polloni, P. T. (1980). The megabenthic fauna in the deep sea south of New England, USA. Mar. Biol. 57: 165-179

Hargreaves, P. M. (1984). The distribution of Decapoda (Crustacea) in the open ocean and near-bottom over an adjacent slope in the northern North-east Atlantic ocean during Autumn 1979. J. mar. biol. Ass. U.K. 64: 829-857

Hargreaves, P. M. (1985). The vertical distribution of decapoda, euphausiacea, and mysidacea at $42^{\circ} \mathrm{N}, 17^{\circ} \mathrm{W}$. Biol. Oceanogr. 3(4): 431-464

Hargreaves, P. M., Ellis, C. J., Angel, M. V. (1984). An assessment of biological processes close to the sea bed in a slope region and its significance to the assessment of sea bed disposal of radioactive waste. IOS Rep. 185

Hopkins, T. L., Baird, R. C. (1975). Net feeding in mesopelagic fishes. Fish. Bull. U.S. 73: 908-914

Hyslop, E. J. (1980). Stomach content analysis. A review of methods and their application. J. Fish Biol. 17: 411-429

Lagardère, J. P. (1972). Recherches sur l'alimentation des Crevettes de la Pente Continentale Marocaine. Tethys 3: $655-675$

Lagardère, J. P. (1976a). Recherches sur l'alimentation des crevettes bathypelagiques du Talus Continentale du Golfe de Gascogne. Revue Trav. Inst. Pêch. marit. 39(2): $213-229$

Lagardère, J. P. (1976b). Recherches sur la distribution verticale et sur l'alimentation des Crustacés Décapodes de la Pente Continentale de l'Atlantique Nord-oriental. Thèse Univ. Aix-Marseille II, Arch. orig. CNRS, 12.237

Lagardère, J. P. (1977). Recherches sur la distribution verticale et sur l'alimentation des crustaces decapodes benthiques de la Pente Continentale du Golfe de Gascogne. Analyse des groupements carcinologiques. Bull. Cent. Etud. Rech. sci. Biarritz 11(4): 367-440

Macpherson, E. (1981). Resource partitioning in a Mediterranean demersal fish community. Mar. Ecol. Prog. Ser. 4: $183-193$

Massuti, M. (1967). Resultados de las pruebas experimentales efectuadas en aguas de Mallorca para la pesca con nasas

This article was submitted to the editor de las gambas de profundidad. Publ. Tec. Junta Est. Pesca 6: $19-85$

Mauchline, J., Gordon, J. D. M. (1985). Trophic diversity in deep-sea fish. J. Fish Biol. 26: 527-535

Pérès, J. M. (1958). Remarques générales sur un ensemble de quinze plongées effectuées avec le bathyscaphe F.N.R.S III. Annls Inst. océanogr., Paris 35: 259-285

Pielou, E. C. (1984). The interpretation of ecological data. John Wiley \& Sons, New York

Rex, M. A. (1977). Zonation in deep-sea gastropods: the importance of biological interactions to rates of zonation. In: Keegan, B. F., Ceidigh, P. O., Boaden, P. J. S. (eds.) Biology of benthic organisms. Pergamon Press, New York. p. $521-530$

Sardà, F., Valladares, F. J. (1990). Gastric evacuation of different foods by Nephrops norvegicus (Crustacea: Decapoda) and estimation of soft tissue ingested, maximum food intake and cannibalism in captivity. Mar. Biol. 104: 25-30

Schoener, T. W. (1970). Nonsynchronous spatial overlap of lizards in patchy habitats. Ecology. 51(3): 408-418

Shannon, C. E., Weaver, W. (1963). The mathematical theory of communication. Univ, of Illinois Press, Urbana

Sokal, R. R., Rohlf, F. J. (1979). Biometria. Principio y métodos estadísticos en la investigación biológica. $\mathrm{H}$. Blume ed., Madrid

Stefanescu, C. (1991). Comunidades ictiológicas demersales del mar catalán (Mediterraneo noroccidental) por debajo de $\operatorname{los} 1000 \mathrm{~m}$ de profundidad. Tesis doctoral, Universidad de Barcelona

Swynnerton, G. H., Worthington, E. B. (1940). Note on the food of fish in Haweswater (Westmoreland). J. Anim. Ecol. 9: $183-187$

Wienberg, R. (1980). On the food and feeding habits of Pendalus borealis Kroyer 1838. Comm. Meer. int. Coun. Explor. Sea. C.M.-ICES/K: 9: 1-25

Wishner, K. F. (1980). The biomass of the deep-sea benthopelagic plankton. Deep Sea Res. 27A: 203-216

Zar, J. H. (1984). Biostatistical analysis. Prentice Hall international Editions, Englewood Cliffs

Zariquiey Alvarez, R. (1968). Crustáceos Decápodos Ibéricos. Invest. pesq. 32: $1-510$

Manuscript first received: August 4, 1992

Revised version accepted: March 2, 1993 